\title{
Solution of Initial Value Prpblem of Bratu - Type Equation using Modifications of Homotopy Perturbation Method
}

\author{
Bothayna S. H. Kashkari \\ Department of Mathematics \\ Faculty of Science, \\ King Abdulaziz University \\ Jeddah, KSA
}

\author{
Sharifa S. Z. Abbas \\ Department of Mathematics \\ Faculty of Science, \\ King Abdulaziz University \\ Jeddah, KSA
}

\begin{abstract}
Homotopy perturbation method (HPM) is an effective method for solving nonlinear differential equations. In this paper, some modifications of this method has been proposed to initial value problem of Bratu - Type model. The combination of Laplace transform and homotopy perturbation (LHPM), the new homotopy perturbation method (NHPM) and Laplace new homotopy perturbation method (LNHPM) are applied, and the solutions are considered as an infinite series that converge rapidly to the exact solutions.
\end{abstract}

\section{Keywords}

Bratu - Type equation, Homotopy perturbation method, Laplace homotopy perturbation method, new homotopy perturbation method, Laplace new homotopy perturbation method

\section{INTRODUCTION}

The Bratu-type equation arise from a simplification of the solid fuel ignition model in thermal combustion theory. This type has many other physical applications, such as chemical reactor theory, radiative heat transfer, electrostatics, fluid mechanics physical applications ranging from chemical reaction theory and nanotechnology to the expansion of universe [1]. Several numerical techniques had been applied to obtain the approximate solution of the Bratu-type problem. For example spline method [2-3], wavelet method [4-6], spectral method [7-10] and Adomain decomposition method [11-14].

In 1992, the homotopy analysis method (HAM) was proposed by Liou [15], some authors used that method and its modifications to solve Bratue - type equation [16-17].

In 1999, J. He presented perturbation technique coupled with the homotopy technique, it was known as HPM [18]. Recently, The homotopy perturbation method and its modification can effectively, easily, and accurately solve a large class of linear and nonlinear, ordinary or partial, deterministic or stochastic differential equations. The approximate solutions converge rapidly to accurate solutions. The method is well-suited to physical problems since it does not require the linearization and other restrictive methods, which may change the problem. The homotopy perturbation method and variational homotopy perturbation method had been applied for Bratue - type problem in [19-20].

In this paper, some modifications of homotopy perturbation method are presented to solve the initial value problem of second order differential equation of Bratu - Type. In section 2, HPM is reviewed. In section 3, the combination of Laplace transform and homotopy perturbation method is presented.
NHPM is reviewed in section 4 . In section 5 , the combination of Laplace transform and new homotopy perturbation method is presented. Finally, in section 6 , the previous methods are applied to second order initial value problems of Bratu Type.

\section{THE HOMOTOPY PERTURBATION METHO}

HPM is combining the classical perturbation technique with the homotopy concept in topology [18].

Consider the nonlinear problem

$A(u)-f(x)=0, \quad x \in \Omega$

with boundary condition

$B\left(u, \frac{d u}{d x}\right)=0, \quad x \in \Gamma$

where $A$ is a general differential operator, $B$ is a boundary operator, $f(x)$ is a known analytic function, $\Gamma$ is the boundary of the domain $\Omega$.

The operator $A$ can be divided into two operators' $L$ and $N$, where $L$ is linear and $N$ is nonlinear, Eq. (0.1) can be rewritten as

$L(u)+N(u)-f(x)=0$

Construct a homotopy $v(x, p): \Omega \times[0,1] \rightarrow \mathbf{R} \quad$ which satisfies

$$
\begin{aligned}
\phi(v, p)= & (1-p)\left[L(v)-L\left(u_{0}\right)\right] \\
& +p[A(v)-f(x)]=0, \quad p \in[0,1], x \in \Omega
\end{aligned}
$$

or

$$
\begin{aligned}
\phi(v, p)= & L(v)-L\left(u_{0}\right)+p L\left(u_{0}\right) \\
& +p[N(v)-f(x)]=0
\end{aligned}
$$

where $p \in[0,1]$ is an embedding parameter, $u_{0}$ is an initial approximation of Eq. (0.1), which satisfies the boundary conditions.

Assume that the solution of Eq. (0.4) can be written as a power series in $p$ : 
$v=\sum_{n=0}^{\infty} p^{n} v_{n}$

Substituting Eq. (0.6) into Eq. (0.5) and collecting terms of the same power of $p$ gives

$$
\begin{array}{ll}
p^{0}: \quad & v_{0}=u_{0} \\
p^{1}: \quad & \left\{\begin{array}{l}
L\left(v_{1}\right)=-L\left(u_{0}\right)-N\left(v_{0}\right)+f(x) \\
B\left(v_{1}, \frac{d v_{1}}{d x}\right)=0
\end{array}\right. \\
p^{2}: \quad\left\{\begin{array}{l}
L\left(v_{2}\right)=-N\left(v_{0}, v_{1}\right) \\
B\left(v_{2}, \frac{d v_{2}}{d x}\right)=0
\end{array}\right. \\
\vdots \begin{array}{l}
L\left(v_{i}\right)=-N\left(v_{0}, v_{1}, \ldots, v_{i-1}\right) \\
B\left(v_{i}, \frac{d v_{i}}{d x}\right)=0
\end{array}
\end{array}
$$

Setting $p=1$, the approximate solution of Eq. (0.1) will be

$u=\lim _{p \rightarrow 1} v=\sum_{i=0}^{\infty} v_{i}$

The series in Eq. (0.11) converges for most cases and it depends upon the nonlinear operator $A(v)$.

\section{LAPLACE HOMOTOPY PERTURBATION METHOD}

LHPM is combining Laplace transform and HPM, it has good stability properties, this method is started by applying Laplace transform on both sides of (0.5), we get

$$
\begin{aligned}
\mathscr{L}\{L(v) & -L\left(u_{0}\right)+p L\left(u_{0}\right) \\
& +p[N(v)-f(x)]\}=0
\end{aligned}
$$

By using the differential property of Laplace transform

$$
\begin{aligned}
\mathscr{L}\{v\}= & \frac{1}{s^{n}}\left\{s^{n-1} v(0)+s^{n-2} v^{\prime}(0)+\cdots+v^{(n-1)}(0)\right. \\
& \left.+\mathcal{L}\left\{L\left(u_{0}\right)-p L\left(u_{0}\right)-p[N(v)-f(x)]\right\}\right\}
\end{aligned}
$$

By applying inverse Laplace transform on both side of Eq. $(0.13)$

$$
\begin{aligned}
v= & \mathcal{L}^{-1}\left\{\frac { 1 } { s ^ { n } } \left\{s^{n-1} v(0)+s^{n-2} v^{\prime}(0)+\cdots+v^{(n-1)}(0)\right.\right. \\
& \left.+\mathcal{L}\left\{L\left(u_{0}\right)-p L\left(u_{0}\right)-p[N(v)-f(x)]\right\}\right\}
\end{aligned}
$$

Rewrite Eq. (0.14) is in the form

$$
\begin{aligned}
\sum_{n=0}^{\infty} p^{n} v_{n}= & \mathcal{L}^{-1}\left\{\frac { 1 } { s ^ { n } } \left\{s^{n-1} v(0)+s^{n-2} v^{\prime}(0)+\cdots\right.\right. \\
& +v^{(n-1)}(0)+\mathcal{L}\left\{L\left(u_{0}\right)-p L\left(u_{0}\right)\right. \\
& \left.\left.\left.-p\left[N\left(\sum_{n=0}^{\infty} p^{n} v_{n}\right)-f(x)\right]\right\}\right\}\right\}
\end{aligned}
$$

$(0.15)$

By equating the terms with identical powers of $p$

$$
\begin{aligned}
& p^{0}: \quad v_{0}= \mathcal{L}^{-1}\left\{\frac { 1 } { s ^ { n } } \left\{s^{n-1} v(0)+s^{n-2} v^{\prime}(0)\right.\right. \\
&\left.\left.+\cdots+v^{(n-1)}(0)+\mathcal{L}\left\{L\left(u_{0}\right)\right\}\right\}\right\} \\
& p^{1}: \quad v_{1}= \mathcal{L}^{-1}\left\{\frac { 1 } { s ^ { n } } \mathcal { Z } \left\{-L\left(u_{0}\right)-\right.\right. \\
& {\left.\left.\left[N\left(v_{0}\right)-f(x)\right]\right\}\right\} } \\
& p^{2}: \quad v_{2}= \mathcal{L}^{-1}\left\{\frac{1}{s^{n}} \mathcal{L}\left\{-\left[N\left(v_{0}, v_{1}\right)\right]\right\}\right\} \\
& \vdots \quad v_{i}= \\
& p^{-1}\left\{\frac{1}{s} \mathfrak{X}\left\{-\left[N\left(v_{0}, v_{1}, \ldots, v_{i-1}\right)\right]\right\}\right\}
\end{aligned}
$$

Assuming that the initial approximation has the form $v(0)=u_{0}=\alpha_{0}, v^{\prime}(0)=\alpha_{1}, \ldots, v^{(n-1)}(0)=\alpha_{n-1}$

\section{NEW HOMOTOPY PERTURBATION METHOD}

For solving Eq. (0.1) by NHPM [21], construct the following homotopy

$$
\begin{aligned}
\phi(v, p)=(1-p)\left[L(v)-u_{0}\right] & \\
+p[A(v)-f(x)]=0, & \\
& p \in[0,1], x \in \Omega
\end{aligned}
$$

or equivalently

$$
\begin{aligned}
\phi(v, p) & =L(v)-u_{0}+p u_{0} \\
& +p[N(v)-f(x)]=0
\end{aligned}
$$

Applying the inverse operator $L^{-1}$ to both sides of Eq. (0.21) gives

$$
\begin{aligned}
& v=L^{-1}\left(u_{0}\right)-p L^{-1}\left(u_{0}\right) \\
& \quad-p L^{-1}[N(v)-f(x)]=0
\end{aligned}
$$

Assume that the initial approximation of Eq. (0.1) has the form

$u_{0}=\sum_{n=0}^{\infty} a_{n} P_{n}(x)$ 
Where $a_{0}, a_{1}, a_{2}, \ldots$ are unknown coefficients which must be computed and $P_{0}, P_{1}, P_{2} \ldots$ are specific functions depending on the problem. Substituting Eq. (0.23) into Eq. (4.3) leads to

$$
\begin{aligned}
\sum_{n=0}^{\infty} p^{n} v_{n} & =L^{-1}\left(\sum_{n=0}^{\infty} a_{n} P_{n}(x)\right)-p L^{-1}\left(\sum_{n=0}^{\infty} a_{n} P_{n}(x)\right) \\
& -p L^{-1}\left[N\left(\sum_{n=0}^{\infty} p^{n} v_{n}\right)-f(x)\right]=0
\end{aligned}
$$

By equating the terms with identical powers of $p$

$$
\begin{aligned}
p^{0}: \quad v_{0}= & L^{-1}\left(\sum_{n=0}^{\infty} a_{n} P_{n}(x)\right) \\
p^{1}: \quad v_{1}= & -L^{-1}\left(\sum_{n=0}^{\infty} a_{n} P_{n}(x)\right) \\
& -L^{-1}\left[N\left(v_{0}\right)-f(x)\right] \\
p^{2}: \quad v_{2}= & -L^{-1}\left[N\left(v_{0}, v_{1}\right)\right] \\
\vdots & \\
p^{i}: \quad v_{i}= & -L^{-1}\left[N\left(v_{0}, v_{1}, \ldots, v_{i-1}\right)\right]
\end{aligned}
$$

If these equations are solved by considering $v_{1}(x)=0$, then $v_{2}(x)=v_{3}(x)=\cdots=0$. Hence the exact solution may be written as

$$
u(x)=v_{0}(x)=L^{-1}\left(\sum_{n=0}^{\infty} a_{n} P_{n}(x)\right)
$$

\section{LAPLACE NEW HOMOTOPY PERTURBATION METHOD}

In LNHPM Laplace transform and NHPM are combined [22], taking Laplace transform on both sides of Eq. (0.21) leads to

$$
\begin{aligned}
\mathscr{L}\{L(v)\}= & \mathscr{L}\left\{u_{0}-p u_{0}\right. \\
& -p[N(v)-f(x)]\}
\end{aligned}
$$

Using the differential property of Laplace transform gives

$$
\begin{aligned}
\mathscr{L}\{v\}= & \frac{1}{s^{n}}\left\{s^{n-1} v(0)+s^{n-2} v^{\prime}(0)+\cdots+v^{(n-1)}(0)\right. \\
& \left.+\mathscr{L}\left\{u_{0}-p u_{0}-p[N(v)-f(x)]\right\}\right\}
\end{aligned}
$$

Applying inverse Laplace transform to both side of Eq. (0.31) leads to

$$
\begin{aligned}
v= & \mathscr{L}^{-1}\left\{\frac { 1 } { s ^ { n } } \left\{s^{n-1} v(0)+s^{n-2} v^{\prime}(0)+\cdots+v^{(n-1)}(0)\right.\right. \\
& \left.\left.+\mathscr{Z}\left\{u_{0}-p u_{0}-p[N(v)-f(x)]\right\}\right\}\right\}
\end{aligned}
$$

By substituting the series of $v, u_{0}$ once can rewrite Eq. $(0.32)$ as

$$
\begin{aligned}
& \sum_{n=0}^{\infty} p^{n} v_{n}=\mathscr{L}^{-1}\left\{\frac { 1 } { s ^ { n } } \left\{s^{n-1} v(0)+s^{n-2} v^{\prime}(0)+\cdots\right.\right. \\
& +v^{(n-1)}(0)+\mathcal{L}\left\{\sum_{n=0}^{\infty} a_{n} P_{n}(x)-p \sum_{n=0}^{\infty} a_{n} P_{n}(x)\right. \\
& \left.\left.\left.-p\left[N\left(\sum_{n=0}^{\infty} p^{n} v_{n}\right)-f(x)\right]\right\}\right\}\right\}
\end{aligned}
$$

By equating the terms with identical powers of $p$

$$
\begin{aligned}
p^{0}: \quad & v_{0}=\mathscr{L}^{-1}\left\{\frac { 1 } { s ^ { n } } \left\{s^{n-1} v(0)+s^{n-2} v^{\prime}(0)\right.\right. \\
& \left.+\cdots+v^{(n-1)}(0)+\mathcal{L}\left\{\sum_{n=0}^{\infty} a_{n} P_{n}(x)\right\}\right\}
\end{aligned}
$$

$(0.34)$

$$
\begin{aligned}
& p^{1}: v_{1}=\mathscr{L}^{-1}\left\{\frac { 1 } { s ^ { n } } \mathscr { L } \left\{-\sum_{n=0}^{\infty} a_{n} P_{n}(x)\right.\right. \\
&\left.\left.\left.-\left[N\left(v_{0}\right)-f(x)\right]\right\}\right\}\right\} \\
& p^{2}: \quad v_{2}=\mathscr{L}^{-1}\left\{-\frac{1}{s^{n}} \mathscr{L}\left\{N\left(v_{0}, v_{1}\right)\right\}\right\}
\end{aligned}
$$

$$
\vdots
$$$$
p^{i}: \quad v_{i}=\mathscr{L}^{-1}\left\{-\frac{1}{s^{n}} \mathscr{L}\left\{N\left(v_{0}, v_{1}, \ldots, v_{i-1}\right)\right\}\right\}
$$

As in NHPM $a_{0}, a_{1}, a_{2}, \ldots$ are computed and solved these equations by considering $v_{1}(x)=0$.

\section{ILLUSTRATIVE EXAMPLE}

Consider the initial value problem of Bratu-Type

$$
\begin{array}{ll}
u^{\prime \prime}-2 e^{u}=0 & 0<x<1 \\
u(0)=u^{\prime}(0)=0 &
\end{array}
$$

The exact solution of above equation is

$$
\begin{aligned}
u(x)= & -2 \ln (\cos x) \\
= & x^{2}+\frac{x^{4}}{6}+\frac{2 x^{6}}{45}+\frac{17 x^{8}}{1260}+\frac{62 x^{10}}{14175} \\
& +\frac{691 x^{12}}{467775}+\cdots
\end{aligned}
$$

To solve Eq. (0.38) by HPM, construct the following homotopy

$$
(1-p)\left[v^{\prime \prime}-u_{0}^{\prime \prime}\right]+p\left[v^{\prime \prime}-2 e^{v}\right]=0, \quad p \in[0,1]
$$


$(0.40)$

By applying Eq. (0.7) - (0.10)

$$
\begin{array}{ll}
p^{0}: & v_{0}=u_{0}=x^{2} \\
p^{1}: & \left\{\begin{array}{l}
v_{1}^{\prime \prime}=-2+2 \sum_{i=0}^{\infty} \frac{v_{0}^{i}}{i !} \\
v_{1}(0)=v_{1}^{\prime}(0)=0
\end{array}\right. \\
p^{2}: \quad\left\{\begin{array}{l}
v_{2}^{\prime \prime}=2 \sum_{i=0}^{\infty} \frac{v_{0}^{i} v_{1}}{i !} \\
v_{2}(0)=v_{2}^{\prime}(0)=0
\end{array}\right.
\end{array}
$$

By solving the differential equations above $v_{0}, v_{1}, v_{2}, \ldots$ are found

$v_{0}(x)=x^{2}$

$v_{1}(x)=\frac{x^{4}}{6}+\frac{x^{6}}{30}+\frac{x^{8}}{168}+\frac{x^{10}}{1080}+\frac{x^{12}}{7920}+\cdots$

$v_{2}(x)=\frac{x^{6}}{90}+\frac{x^{8}}{140}+\frac{103 x^{10}}{37800}+\frac{97 t^{12}}{124740}+\cdots$

$v_{3}(x)=\frac{x^{8}}{2520}+\frac{x^{10}}{1400}+\frac{659 x^{12}}{1247400}+\cdots$

And once can get

$$
\begin{aligned}
v(x)= & \sum_{i=0}^{\infty} v_{i}(x)=x^{2}+\frac{x^{4}}{6}+\frac{2 x^{6}}{45}+\frac{17 x^{8}}{1260} \\
& +\frac{62 x^{10}}{14175}+\frac{691 x^{12}}{467775}+\cdots
\end{aligned}
$$

Which converge to the exact solution in Eq. (0.39).

To solve Eq. (0.38) by LHPM, apply Laplace transform to both sides of Eq. (0.40)

$$
\mathcal{L}\left\{v^{\prime \prime}-u_{0}^{\prime \prime}+p u_{0}^{\prime \prime}-2 p e^{v}\right\}=0
$$

By applying inverse Laplace transform

$$
v=\mathscr{L}^{-1}\left\{\frac{1}{s^{n}}\left\{\mathscr{L}\left\{u_{0}^{\prime \prime}-p u_{0}^{\prime \prime}-p e^{v}\right\}\right\}\right.
$$

Equating the terms with identical powers of $p$ as Eq. (0.16) (0.37) gives

$$
\begin{aligned}
p^{0}: \quad v_{0} & =\mathscr{L}^{-1}\left\{\frac{1}{s^{n}} \mathscr{L}\{2\}\right\}=x^{2} \\
p^{1}: \quad v_{1} & =\mathscr{L}^{-1}\left\{\frac{1}{s^{n}} \mathscr{L}\left\{-2+2 \sum_{i=0}^{\infty} \frac{v_{0}^{i}}{i !}\right\}\right\} \\
& =\frac{x^{4}}{6}+\frac{x^{6}}{30}+\frac{x^{8}}{168}+\frac{x^{10}}{1080}+\cdots \\
p^{2}: \quad v_{2} & =\mathscr{L}^{-1}\left\{\frac{1}{s^{n}} \mathcal{L}\left\{2 \sum_{i=0}^{\infty} \frac{v_{0}{ }^{i} v_{1}}{i !}\right\}\right\} \\
& =\frac{x^{6}}{90}+\frac{x^{8}}{140}+\frac{103 x^{10}}{37800}+\cdots
\end{aligned}
$$

\section{:}

Thus

$$
\begin{aligned}
v(x)= & \sum_{i=0}^{\infty} v_{i}(x)=x^{2}+\frac{x^{4}}{6}+\frac{2 x^{6}}{45}+\frac{17 x^{8}}{1260} \\
& +\frac{62 x^{10}}{14175}+\frac{691 x^{12}}{467775}+\cdots
\end{aligned}
$$

Which converge to the exact solution in Eq. (0.39).

For solving Eq. (0.38) by NHPM, consider the homotopy

$(1-p)\left[v^{\prime \prime}-u_{0}\right]+p\left[v^{\prime \prime}-2 e^{v}\right]=0$

By applying the inverse operator $L^{-1}=\int_{0}^{x} \int_{0}^{t}() d r d$.$t to both$ sides of Eq. (0.48)

$$
\begin{aligned}
v(x)= & v(0)+v^{\prime}(0)+\int_{0}^{x} \int_{0}^{t} u_{0}(r) d r d t \\
& -p \int_{0}^{x} \int_{0}^{t} u_{0}(r) d r d t+2 p \int_{0}^{x} \int_{0}^{t} e^{v(r)} d r d t
\end{aligned}
$$

By substituting the series of $v, u_{0}$ once can rewrite Eq. (0.49) as

$$
\begin{aligned}
\sum_{n=0}^{\infty} p^{n} v_{n} & =\int_{0}^{x} \int_{0}^{t} \sum_{n=0}^{\infty} a_{n} r^{n} d r d t-p \int_{0}^{x} \int_{0}^{t} \sum_{n=0}^{\infty} a_{n} r^{n} d r d t \\
& +2 p \int_{0}^{x} \int_{0}^{t} \sum_{0=0}^{\infty} \frac{v^{i}(r)}{i !} d r d t
\end{aligned}
$$

Equating the terms with identical powers of $p$ as Eq. (0.25) $(0.28)$ gives 


$$
\begin{aligned}
& p^{0}: \quad v_{0}=\int_{0}^{x} \int_{0}^{t} \sum_{n=0}^{\infty} a_{n} r^{n} d r d t \\
& =\frac{x^{2} a_{0}}{2}+\frac{x^{3} a_{1}}{6}+\frac{x^{4} a_{2}}{12}+\frac{x^{5} a_{3}}{20} \\
& +\frac{x^{6} a_{4}}{30}+\frac{x^{7} a_{5}}{42}+\frac{x^{8} a_{6}}{56}+\cdots \\
& p^{1}: \quad v_{1}=-\int_{0}^{x} \int_{0}^{t} \sum_{n=0}^{\infty} a_{n} r^{n} d r d t \\
& +2 \int_{0}^{x} \int_{0}^{t} \sum_{n=0}^{\infty} \frac{v_{0}^{i}(r)}{i !} d r d t \\
& =x^{2}\left(1-\frac{a_{0}}{2}\right)-\frac{x^{3} a_{1}}{6}+x^{4}\left(\frac{a_{0}}{12}-\frac{a_{2}}{12}\right) \\
& +x^{5}\left(\frac{a_{1}}{60}-\frac{a_{3}}{20}\right) \\
& +x^{6}\left(\frac{a_{0}^{2}}{120}+\frac{a_{2}}{180}-\frac{a_{4}}{30}\right) \\
& +x^{7}\left(\frac{a_{0} a_{1}}{252}+\frac{a_{3}}{420}-\frac{a_{5}}{42}\right) \\
& +x^{9}\left(\frac{a_{0}^{2} a_{1}}{1728}+\frac{a_{1} a_{2}}{2592}+\frac{a_{0} a_{3}}{1440}+\frac{a_{5}}{1512}\right) \\
& +x^{11}\left(\begin{array}{l}
\frac{a_{0}^{3} a_{1}}{15840}+\frac{a_{1}^{3}}{71280}+\frac{a_{0} a_{1} a_{2}}{7920}+\frac{a_{0}^{2} a_{3}}{8800} \\
+\frac{a_{2} a_{3}}{13200}+\frac{a_{1} a_{4}}{9900}+\frac{a_{0} a_{5}}{4620}
\end{array}\right) \\
& +x^{8}\left(\frac{a_{0}^{3}}{1344}+\frac{a_{1}^{2}}{2016}+\frac{a_{0} a_{2}}{672}+\frac{a_{4}}{840}-\frac{a_{6}}{56}\right)+\cdots
\end{aligned}
$$

Vanishing $v_{1}(x)$ lets the coefficients $a_{n}(n=0,1,2, \ldots)$ to take the following values

$$
\begin{aligned}
& a_{0}=2, a_{2}=2, a_{4}=\frac{4}{3}, a_{6}=\frac{34}{45}, \cdots \\
& a_{1}=a_{3}=a_{5}=\cdots=0
\end{aligned}
$$

Hence the solution of Eq. (0.38) is written as

$u(x)=v_{0}(x)=x^{2}+\frac{x^{4}}{6}+\frac{2 x^{6}}{45}+\frac{17 x^{8}}{1260}+\cdots$

And this in the limit of infinity many terms, yields the exact solution in Eq. (0.39).

For solving Eq. (0.38) by LNHPM, apply Laplace transform to both sides of Eq. (0.48)

$$
\begin{aligned}
\mathscr{L}\{v\}= & \frac{1}{s^{2}}\left\{s v(0)+v^{\prime}(0)\right. \\
& \left.+\mathscr{L}\left\{u_{0}-p u_{0}+2 p e^{v}\right\}\right\}
\end{aligned}
$$

By applying inverse Laplace transform

$v=\mathscr{L}^{-1}\left\{\frac{1}{s^{2}} \mathscr{L}\left\{u_{0}-p u_{0}+2 p e^{v}\right\}\right\}$

By substituting the series of $v, u_{0}$ once can rewrite Eq. $(0.55)$ as

$$
\begin{aligned}
\sum_{n=0}^{\infty} p^{n} v_{n} & =\mathscr{L}^{-1}\left\{\frac { 1 } { s ^ { 2 } } \mathcal { L } \left\{\sum_{n=0}^{\infty} a_{n} r^{n}-p \sum_{n=0}^{\infty} a_{n} r^{n}\right.\right. \\
& \left.\left.+2 p \sum_{n=0}^{\infty} \frac{v^{i}(r)}{i !}\right\}\right\}
\end{aligned}
$$

Equating the terms with identical powers of $p$ leads to

$$
\begin{aligned}
p^{0}: \quad & v_{0}=\mathcal{L}^{-1}\left\{\frac { 1 } { s ^ { 2 } } \left\{s^{2} v(0)+v^{\prime}(0)\right.\right. \\
& \left.+\mathcal{L}\left\{\sum_{n=0}^{\infty} a_{n} x^{n}\right\}\right\}=\mathcal{L}^{-1}\left\{\frac{1}{s^{2}} \mathcal{L}\left\{\sum_{n=0}^{\infty} a_{n} x^{n}\right\}\right\} \\
& =\frac{x^{2} a_{0}}{2}+\frac{x^{3} a_{1}}{6}+\frac{x^{4} a_{2}}{12}+\frac{x^{5} a_{3}}{20}+\frac{x^{6} a_{4}}{30} \\
& +\frac{x^{7} a_{5}}{42}+\frac{x^{8} a_{6}}{56}+\cdots \\
p^{1}: \quad & v_{1}=\mathcal{L}^{-1}\left\{\frac{1}{s^{2}} \mathcal{L}\left\{-\sum_{n=0}^{\infty} a_{n} x^{n}-2 \sum_{n=0}^{\infty} \frac{v_{0}^{i}}{i !}\right\}\right\} \\
& =\frac{1}{2} x^{2}\left(2-a_{0}\right)-\frac{x^{3} a_{1}}{6}+\frac{1}{12} x^{4}\left(a_{0}-a_{2}\right) \\
& +\frac{1}{60} x^{5}\left(a_{1}-3 a_{3}\right) \\
& +\frac{1}{360} x^{6}\left(3 a_{0}^{2}+2 a_{2}-12 a_{4}\right) \\
& +x^{7} \frac{\left(5 a_{0} a_{1}+3 a_{3}-30 a_{5}\right)}{1260} \\
& +x^{8} \frac{\left(15 a_{0}^{3}+10 a_{1}^{2}+30 a_{0} a_{2}+24 a_{4}-360 a_{6}\right)}{20160} \\
& +x^{9} \frac{\left(15 a_{0}^{2} a_{1}+10 a_{1} a_{2}+18 a_{0} a_{3}\right)}{25920} \cdots
\end{aligned}
$$

Vanishing $v_{1}(x)$ lets the coefficients $a_{n}(n=0,1,2, \ldots)$ to take the following values 
$a_{0}=2, a_{2}=2, a_{4}=\frac{4}{3}, a_{6}=\frac{34}{45}, \ldots$

$a_{1}=a_{3}=a_{5}=\cdots=0$

Therefore, the solution of Eq. (0.38) can be written as

$u(x)=v_{0}(x)=x^{2}+\frac{x^{4}}{6}+\frac{2 x^{6}}{45}+\frac{17 x^{8}}{1260}+\cdots$

Which converge to the exact solution in Eq. (0.39).

\section{CONCLUSION}

In this paper, some modifications of homotopy perturbation method have used for solving second order initial value problem of Bratu type. It should be mentioned the results of the methods exhibit excellent agreement with the exact solution. The computations corresponding to the examples have been performed using Mathematica.

\section{REFERENCES}

[1] S. Hichar, et al., "Application of nonlinear Bratu's equation in two and three dimension to electrostatics," Reports on mathematical physics, Vol. 76, No. 3, pp. 283 $-290,2015$

[2] C. Hikmet, et al., "B-spline method for solving Bratu's problem," International Journal of Computer Mathematics, Volume 87, No. 8, pp. 1885 - 1891, 2010.

[3] R. Jalilian, "Non-polynomial spline method for solving Bratu's problem," Computer Physics Communications, Volume 181, No. 11, pp. 1868 - 1872, 2010.

[4] C. Yang and J. Hou, "Chebyshev wavelets method for solving Bratu's problem", Doc 391, Boundary Value Problems, Volume 2013, No. 1, pp. 142, 2013.

[5] A. Kazemi, et al., "An efficient approach for solving nonlinear Troesch's and Bratu's problems by wavelet analysis method," Mathematical Problems in Engineering, Volume 2013, 2013.

[6] G. Venkatesh, et al., "The Legendre wavelet method for solving initial value problems of Bratu-type," Computers \& Mathematics with Applications, Volume 63, No. 8, pp. $1287-1295,2012$.

[7] E. Doha, et al., "Efficient Jacobi-Gauss collocation method for solving initial value problems of Bratu type," Computational Mathematics and Mathematical Physics, Volume 53 No. 9, pp. 1292 - 1302, 2013.

[8] M. Abd-Elhameed, et al., "New Spectral Second Kind Chebyshev Wavelets Algorithm for Solving Linear and Nonlinear Second-Order Differential Equations Involving Singular and Bratu Type Equations," Abstract and Applied Analysis, Volume 2013, pp. 1 - 9, 2013.

[9] M. Abd-Elhameed, et al., "A novel operational matrix method based on shifted Legendre polynomials for solving second-order boundary value problems involving singular, singularly perturbed and Bratu-type equations," Mathematical Sciences, Vol. 9, No. 2, pp. 93 - 102, 2015.

[10] P. John, "One-point pseudospectral collocation for the one-dimensional Bratuequation," Applied Mathematics and Computation, Vol. 217, No. 12, pp. 5553 - 5565, 2011.

[11] M. Syam and H. Abdelrahem, ("OA59efficient method for solving Bratu equations," Applied Mathematics and Computation, Vol. 176, No. 2, pp. 704 - 713, 2006.

[12] A. Wazwaz, "Adomian decomposition method for a reliable treatment of the Bratu-type equations," Applied Mathematics and Computation, Vol. 166, No. 3, pp. 652663, 2005.

[13] A. Vahidi and M. Hasanzade, "Restarted Adomian's Decomposition Method for the Bratu-Type Problem," Applied Mathematical Sciences, Vol. 6, No. 10, pp. 479 $-486,2012$.

[14] O. Samuel, et al., "A New Result On Adomian Decomposition Method for Solving Bratu's Problem," Mathematical Theory and Modeling Vol.3, No.2, 2013.

[15] S. Liao, "Homotopy Analysis Method in Nonlinear Differential Equations," Higher Education Press, Beijing and Springer-Verlag Berlin Heidelberg, 2012.

[16] N. Hany and S. Mourad , "Analytic approximate solution for the Bratu's problem by optimal homotopy analysis method," Communications in Numerical Analysis, Volume 2013, pp. 1 - 14, 2013.

[17] M. Darwish and B. Kashkari, "Numerical solutions of second order initial value problems of Bratu-type via optimal homotopy asymptotic method," American Journal of Computational Mathematics, Vol. 4, No. 2, pp. $47-54,2014$.

[18] J. He, "Homotopy perturbation technique," Comput. Methods Appl. Mech. Engrg. Vol. 178, pp. 257 - 262, 1999.

[19] X. Feng, et al., "Application of homotopy perturbation method to the Bratu - Type equations," Topological Methods in Nonlinear Analysis Journal of Juliusz Schauder Center, Vol. 31, pp. 243 - 252, 2008.

[20] A. Ezekiel, "New Improved Variational Homotopy Perturbation Method for Bratu-Type Problems," American Journal of Computational Mathematics, Vol. 3, No. 2, pp. $110-113,2013$.

[21] H. Aminikhah H. and M. Hemmatnezhad, "An efficient method for quadratic Riccati differential equation," Commun Nonlinear Sci Numer Simulat 15, pp. 835-839, 2010.

[22] H. Aminikhah, "The combined Laplace transform and new homotopy perturbation methods for stiff systems of ODEs," Applied Mathematical Modelling, Vol. 36, pp.3638-3644, 2012 